\title{
Research on the Situations of Polyploid Formation of Lavender Induced by Colchicine
}

\author{
Xue Chunmei $^{1, \text { a }}$, Ren Guoli ${ }^{2, b}$, Zhao Chenchen ${ }^{2, c}$, Zhang Yuehua ${ }^{2, \text { d,* }}$ \\ ${ }^{1}$ Jiamusi University College of Life Sciences, Heilongjiang Province 154007, China \\ ${ }^{2}$ Jiamusi University College of Science, Heilongjiang Province 154007, China \\ ajmsxcm@126.com, ${ }^{b} 312541669 @ q q . c o m,{ }^{c}$ zhaochenchen89@126.com \\ ${ }^{d, *}$ Corresponding Author: zhangyaohua_2008@163.com
}

Keywords: lavender, mutation frequency, merge rate, polyploidy

\begin{abstract}
To explore the optimum concentration and treatment time of colchicine to induce chromosome doubling, the optimal conditions of polyethylene glycol-induced protoplast fusion molecular weight, working concentration and induction time, using orthogonal and single factor, the screening test of high quality characters of lavender polyploidy were carried out. Using colchicine method combined with PEG method for lavender polyploid induction, polyploid plants with excellent traits were selected. Among them, the protoplast fusion rate of PEG with a molecular weight of 6000Da was $19.6 \%$ after being induced at $40 \%$ for 25 min. Lavender explants were treated with colchicine MS induction medium at a concentration of $0.2 \%$ for $48 \mathrm{~h}$ and the mutagenicity frequency reached $60 \%$. The results showed that the method of polyploid induction of lavender can quickly realize breeding of polyploid varieties of lavender and shorten the breeding cycle, and provide basic data theoretical basis for further study on the industrialized production of lavender and highly oil-resistant polyploid plants.
\end{abstract}

\section{Introduction}

Lavandula angustifolia is Labiatae lavender of herb, native to the western Mediterranean ${ }^{[1]}$. Sap is an effective sebum regulator, extracted essential oil with calming nerves, depression, inhibited of bacterial growth ${ }^{[2]}$. In recent years, lavender has received widespread attention as a garden ornamental plant, and its market has a bright future. At present, the main varieties of lavender in China rely on the introduction of foreign countries. Due to constraints of environment and geographical location and other factors, gradually high light germination rate of seeds and the decline of quality of Lavender essential oil restrict the rapid development of lavender industry chain. Finding lavender efficient and scalable research methods are imperatively ${ }^{[3]}$. Policy breeding methods can improve the genetic traits of plants and expand their genetic resources ${ }^{[4]}$. In this experiment, lavender was used as a material for lavender polyploid induction. To investigate the effects of colchicine concentration and treatment time on the induced polyploid mutation rate.In order to cultivate polyploid plants with excellent traits, then increase the yield of lavender oil for Heilongjiang Province to replace the traditional crop varieties and ease the shortage of species to provide the basic research status quo ${ }^{[5]}$. 


\section{Materials and Methods}

\subsection{Material}

Material: Callus, Lavender growth of tissue culture seedlings, the sterile seedlings cut into stem segments containing two stems, callus are crushed to $1 \mathrm{~mm}^{3}$

Preparation of the main reagent: MS solid medium, MS liquid medium, $0.1 \%$ MES, 0.6 $\mathrm{mol} \cdot \mathrm{L}^{-1}$ mannitol

\subsection{Method}

\subsubsection{Protoplast purification}

Preparation of colchicine inducer: Colchicine inducer was formulated at a concentration of $0.01 \%, 0.05 \%, 0.1 \%, 0.2 \%, 0.3 \%, 0.4 \%$ and $0.5 \%$ by $8.5 \mathrm{~g} \cdot \mathrm{L}^{-1} \mathrm{NaCl}$ in $4{ }^{\circ} \mathrm{C}$ refrigerator shading backup

Preparation of polyethylene glycol fusing agent: $0.1 \mathrm{M}$ sucrose, $0.16 \mathrm{mmol} \cdot \mathrm{L}^{-1} \mathrm{KH}_{2} \mathrm{PO}_{4}, 15.0$ $\mathrm{mmol} \cdot \mathrm{L}^{-1} \mathrm{CaCl}_{2} \cdot 2 \mathrm{H}_{2} \mathrm{O}, 10 \%-50 \%$ PEG X( W:V, X=4000, 6000), 15\% DMSO, The pH is between 5.3 and 5.8

High $\mathrm{Ca}^{2+}$ and $\mathrm{pH}$ solution: $300 \mathrm{mmol} \cdot \mathrm{L}^{-1}$ glucose, $60 \mathrm{mmol} \cdot \mathrm{L}^{-1} \mathrm{CaCl}_{2} \cdot 2 \mathrm{H}_{2} \mathrm{O}, 60 \mathrm{mmol} \cdot \mathrm{L}^{-1}$ glycine-sodium hydroxide buffer, $\mathrm{pH} 10.5$.

Preparation of CPW lotion: $0.05 \mathrm{~mol} \cdot \mathrm{L}^{-1} \mathrm{CaCl}_{2} \cdot 2 \mathrm{H}_{2} \mathrm{O}$ and $0.1 \% \mathrm{MES}$

Preparation of enzyme solution: CPW solution was added $1.0 \%$ peptidase, $1.5 \%$ cellulase and $0.6 \mathrm{~mol} \cdot \mathrm{L}^{-1}$ mannitol, $\mathrm{pH}$ 5.7. After mixing enzyme solution, centrifuged at low temperature of 1 $000 \mathrm{r} \cdot \mathrm{min}^{-1}$ for $20 \mathrm{~min}$. The supernatant was aspirated with a sterile syringe and filtered through a $0.22 \mu \mathrm{m}$ sterile filter to a sterile bottle and sealed for later use.

Protoplast preparation:The selected callus was sterilized with sterile forceps to a size of $1 \mathrm{~mm}^{3}$ in a sterile clean bench and the prepared test material was placed in a sterile flask containing the enzyme solution in a 1: 10 ratio. The flask was placed at a speed of $160 \mathrm{r} \cdot \mathrm{min}^{-1} 25{ }^{\circ} \mathrm{C}$ constant temperature shaker dark shock digestion $12 \mathrm{~h}$ to free protoplasts.

Protoplast purification: Enzymatic hydrolysis of the mixed enzyme solution in a clean bench by 200 mesh stainless steel mesh filter to the residue, ice bath conditions, $1000 \mathrm{r} \cdot \mathrm{min}^{-1}$ centrifugal 20 min, the lower sediment is protoplast crude extract. The crude extract of lavender protoplast was added CPW lotion to $10 \mathrm{~mL}, 1000 \mathrm{r} \cdot \mathrm{min}^{-1}$ centrifugation $20 \mathrm{~min}$, the supernatant was discarded, the above steps were repeated 3 times, with protoplast broth washing 1 . After centrifugation, precipitate is purification protoplasts.

\subsubsection{Colchicine induction method}

Preparation of polyploidy: The tissue culture seedlings of lavender were cut and the stems with uniform stem and two stem segments were cut and inoculated into polyploid induction medium containing different concentrations of colchicine (MS $+1 \mathrm{mg} \cdot \mathrm{L}^{-1} 6-\mathrm{BA}+2.0 \%$ DMSO ), 15 stems were processed and transferred to subculture medium (MS $+1 \mathrm{mg} \cdot \mathrm{L}^{-1} 6-\mathrm{BA}+0.3 \mathrm{mg} \cdot \mathrm{L}^{-1} \mathrm{IAA}$ ) for 24, 48 and 72 hours.After 30 days of culture, the cells were transferred to the subculture medium for further cultivation, repeatedly cultured for several times, homozygotes cultured, and the chimeras were removed to obtain purified polyploid seedlings, and the stable plants with obvious variation were selected for identification. 


\subsubsection{PEG binding high $\mathrm{Ca}^{2+}$ and $\mathrm{pH}$ fusion method}

Preparation of fused cells: Under sterile environment, $1.0 \mathrm{~mL}$ suspension of the purified protoplast was placed in a $10.0 \mathrm{~mL}$ tube of centrifuge and allowed to stand for $8 \mathrm{~min}$ to form a thin layer of protoplast. After the same amount of PEG solution and $2.0 \mathrm{~mL}$ of high $\mathrm{Ca}^{2+}$ and high $\mathrm{pH}$ solution were added. Through5, 10, 15, 20, 25 and 30 min after the addition of CPW washing solution to $10.0 \mathrm{~mL}$, washed three times $\left(1000 \mathrm{r} \cdot \mathrm{min}^{-1}, 20 \mathrm{~min}\right)$, then the culture medium was washed once, finally with the culture fluid suspension was suspended, take $2 \mathrm{~mL}$ suspension in petri dishes for shallow liquid culture.

Identification and counting of fused cells: Take $20 \mu \mathrm{L}$ fusion protoplast drop on the cell count plate, microscopic observation. According to the TTC into the protoplast and mitochondrial succinate dehydrogenase reaction to generate red formazan, and dead cells due to dehydrogenase activity decreased, it will not produce the principle of color changes to identify the dynamic protoplasts. The total number of protoplasts and viable protoplasts were counted several times. All the data were processed by SPSS 16.60. The fusion rate was calculated, see formula (1).

$$
\text { Fusion rate } \%=\frac{\text { Hybrid cells }}{\text { The total number of cells }} \times 100 \%
$$

\section{Result Analyses}

\subsection{Effect of colchicine on inducing variation of lavender}

The results showed that, variation rate changes with the change of concentrationof colchicine and treatment time, the concentration of colchicine increased, the treatment time prolonged, the doubling rate increased, but the mortality of lavender explants also increased relatively0.2\% to $0.3 \%$ colchicine treatment 48 to 72 hours produce relatively good effect, while the $0.2 \%$ colchicine treatment of $48 \mathrm{~h}$ mutation rate up to $60 \%, 0.5 \%$ colchicine treatment lethal rate is too high, Obvious toxic effects, less than $0.05 \%$ mutagenesis rate is almost zero, so with $0.2 \%$ colchicine treatment 48h lavender induction effect is significant (see Table 1). 
Table 1. Changes of polyploid plants induced by colchicine at different concentrations and different treatment times

\begin{tabular}{|c|c|c|c|c|c|c|}
\hline colchicine & $\begin{array}{l}\text { processing } \\
\text { time }\end{array}$ & $\begin{array}{r}\text { number } \\
\text { of samples }\end{array}$ & $\begin{array}{l}\text { number of } \\
\text { deaths }\end{array}$ & mortality & variation number & variation rate \\
\hline \multirow{3}{*}{0.01} & 24 & 15 & 0 & 0 & 0 & 0 \\
\hline & 48 & 15 & 0 & 0 & 0 & 0 \\
\hline & 72 & 15 & 2 & 13.33 & 0 & 0 \\
\hline \multirow{3}{*}{0.05} & 24 & 15 & 0 & 0 & 0 & 0 \\
\hline & 48 & 15 & 2 & 13.33 & 0 & 0 \\
\hline & 72 & 15 & 1 & 6.67 & 1 & 6.67 \\
\hline \multirow{3}{*}{0.1} & 24 & 15 & 1 & 6.67 & 0 & 0 \\
\hline & 48 & 15 & 1 & 6.67 & 1 & 6.67 \\
\hline & 72 & 15 & 2 & 13.33 & 0 & 0 \\
\hline \multirow{3}{*}{0.2} & 24 & 15 & 1 & 6.67 & 2 & 13.33 \\
\hline & 48 & 15 & 1 & 6.67 & 9 & 60 \\
\hline & 72 & 15 & 2 & 13.33 & 6 & 40 \\
\hline \multirow{3}{*}{0.3} & 24 & 15 & 1 & 6.67 & 2 & 13.33 \\
\hline & 48 & 15 & 3 & 20 & 7 & 46.67 \\
\hline & 72 & 15 & 4 & 26.67 & 7 & 46.67 \\
\hline \multirow{3}{*}{0.4} & 24 & 15 & 3 & 20 & 3 & 20 \\
\hline & 48 & 15 & 8 & 53.33 & 5 & 33.33 \\
\hline & 72 & 15 & 13 & 86.67 & 2 & 13.33 \\
\hline \multirow{3}{*}{0.5} & 24 & 15 & 9 & 60 & 5 & 33.33 \\
\hline & 48 & 15 & 15 & 100 & 0 & 0 \\
\hline & 72 & 15 & 15 & 100 & 0 & 0 \\
\hline
\end{tabular}

\subsection{Effect of PEG on the protoplast fusion of lavender}

When the molecular weight of PEG is 4000Da, the PEG concentration is increased, the incubation time is prolonged and the fusion rate is gradually increased, but the fusion rate is lower than the PEG with the molecular weight of 6000Da at the same time and concentration, and the viability of protoplasts is decreased. When the molecular weight of PEG was $6000 \mathrm{Da}$, the PEG concentration increased, the incubation time prolonged and the fusion rate increased gradually. The fusion rate at each treatment time was the highest when the PEG concentration was $40 \%$, and the fusion rate reached $19.6 \%$. Protoplasts shrinkage, volume decrease and color change were observed after 30min.The phenomenon indicated that, when molecular weight of PEG is 6000Da, the concentration is $40 \%$ and the treatment time is $25 \mathrm{~min}$, the fusion effect is obvious (Table 2).

Table 2. Effect of different PEG molecular weight, concentration and incubation time on the fusion rate of lavender protoplasts

\begin{tabular}{ccccccccccc}
\hline & \multicolumn{10}{c}{ Fusion rate } \\
\cline { 2 - 11 } & $10 \%$ & $20 \%$ & $30 \%$ & $40 \%$ & $50 \%$ & $10 \%$ & $20 \%$ & $30 \%$ & $40 \%$ & $50 \%$ \\
& 2.5 & 3.5 & 5.2 & 6.9 & 8.2 & 3.6 & 4.5 & 5.8 & 7.9 & 7.6 \\
$5 \mathrm{~min}$ & 3.4 & 5.2 & 6.8 & 7.8 & 9.4 & 4.2 & 5.7 & 7.3 & 9.2 & 8.9 \\
$10 \mathrm{~min}$ & 4.2 & 6.7 & 9.4 & 10.6 & 11.6 & 5.7 & 7.2 & 9.8 & 12.4 & 11.4 \\
$15 \mathrm{~min}$ & 6.3 & 7.9 & 10.2 & 11.4 & 12.2 & 7.3 & 8.3 & 10.4 & 18.8 & 13.6 \\
$20 \mathrm{~min}$ & 7.5 & 8.4 & 10.8 & 12.3 & 12.9 & 8.1 & 8.9 & 11.3 & 19.6 & 14.2 \\
$25 \mathrm{~min}$ & 8.2 & 9.3 & 11.4 & 12.8 & 13.4 & 8.8 & 9.6 & 12.6 & 17.8 & 14.8 \\
$30 \mathrm{~min}$ & & $1000 \mathrm{Da}$ &
\end{tabular}




\section{Discussion and conclusion}

With $0.2 \%$ to $0.3 \%$ of colchicine on lavender tissue culture induction treatment 48 to $72 \mathrm{~h}$ can produce polyploidy. $0.2 \%$ colchicine treatment $48 \mathrm{~h}$ mutagenesis rate up to $60 \%$, but many polyploid is a chimera, so requires subculture for separation. The fusion rate of protoplasts with molecular weight of $6000 \mathrm{Da}$ and $40 \%$ PEG at $25 \mathrm{~min}$ could reach $19.6 \%$.The method of colchicine combined with PEG method was used to induce polyploidy of lavender to screen polyploid plants with excellent traits, which provided theoretical basis and technical support for further research on lavender and industrialized production of high oil-resistant polyploid plants, Can alleviate the shortage of new varieties of local research in Heilongjiang Province. However, there are problems of browning and vitrification in the process of cultivation, which needs further study.

\section{Acknowledgments}

Heilongjiang Provincial Nature Fund Project (C2017064); Jiamusi University Science research Project: JMSUJCMS2016-015.

\section{References:}

[1] Pirali-Kheirabadi, Khodadad, and Jaime A. Teixeira da Silva. "Lavandula angustifolia essential oil as a novel and promising natural candidate for tick (Rhipicephalus (Boophilus) annulatus) control." Experimental Parasitology 126.2 (2010): 184-186.

[2] Verma, Ram S., et al. "Essential oil composition of Lavandula angustifolia Mill. cultivated in the mid hills of Uttarakhand, India." Journal of the serbian chemical society 75.3 (2010): 343-348.

[3] Lew, Marta. Modeling supply chain benefits of efficient assortment. Diss. Massachusetts Institute of Technology, 2010.

[4] Tester M, Langridge P. Breeding technologies to increase crop production in a changing world [J]. Science, 2010, 327(5967): 818-822.

[5] Coelho N, Gonçalves S, González-Benito M E, et al. Establishment of an in vitro propagation protocol for Thymus lotocephalus, a rare aromatic species of the Algarve (Portugal)[J]. Plant growth regulation, 2012, 66(1): 69-74. 